\title{
e-Phaïstos
}

e-Phaïstos

Revue d'histoire des techniques / Journal of the history

of technology

III-1 | 2014

Varia

\section{Une épée d'armes pour le combat singulier en armure}

A arming sword for single combat in armour

\section{Daniel JAQUET}

\section{(2) OpenEdition}

Journals

Édition électronique

URL : http://journals.openedition.org/ephaistos/589

DOI : 10.4000/ephaistos.589

ISSN : 2552-0741

Éditeur

IHMC - Institut d'histoire moderne et contemporaine (UMR 8066)

Édition imprimée

Date de publication : 1 juin 2014

Pagination : 74-82

ISSN : 2262-7340

Référence électronique

Daniel JAQUET, « Une épée d'armes pour le combat singulier en armure », e-Phaïstos [En ligne],

III-1 | 2014, mis en ligne le 18 novembre 2016, consulté le 01 mai 2019. URL : http://

journals.openedition.org/ephaistos/589 ; DOI : 10.4000/ephaistos.589

Tous droits réservés 


\section{Une épée d'armes pour le combat singulier en armure.}

Daniel Jaquet

Université de Genève

Faculté des Lettres

Département d'histoire générale
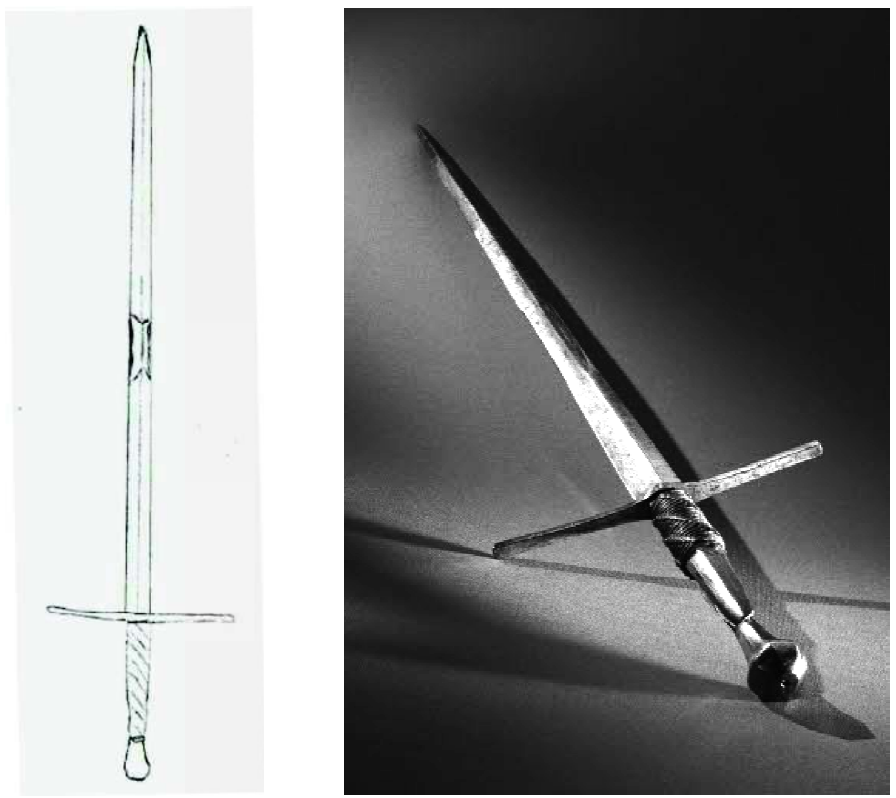

Figures 1 et 2 : Croquis de l'auteur et photographie de l'objet. (C) Stadtmuseum, Wien, HMW 126006

Cette épée portant la marque du loup de Passau (voir fig. 1 et 2), datée des années 1480 est conservée dans les dépôts du Musée de la ville de Vienne. Entre la garde droite et le pommeau en poire renversée à facettes, les deux plaquettes de bois formant la poignée, reliées par du tissu et de la corde peints en rouge, sont originales. Sa lame en alliage ferreux à section carrée de $74 \mathrm{~cm}$ de long sur $3.5 \mathrm{~cm}$ de large à sa base comporte deux particularités : d'une part, la lame est asymétrique, un tranchant étant donc significativement plus important; d'autre part, en son milieu, une partie de $14 \mathrm{~cm}$ de long est ergonomiquement différente de l'ensemble, formée par une concentration de la matière.

De par son caractère relativement unique ${ }^{1}$, cette arme est sortie dans au moins trois expositions au cours de ces cinq dernières années. La littérature ${ }^{2}$ qui l'accompagne diffère néanmoins au sujet de la présence de cette partie spécifique au milieu de la lame, qui rend l'utilisation du tranchant de l'arme problématique. 


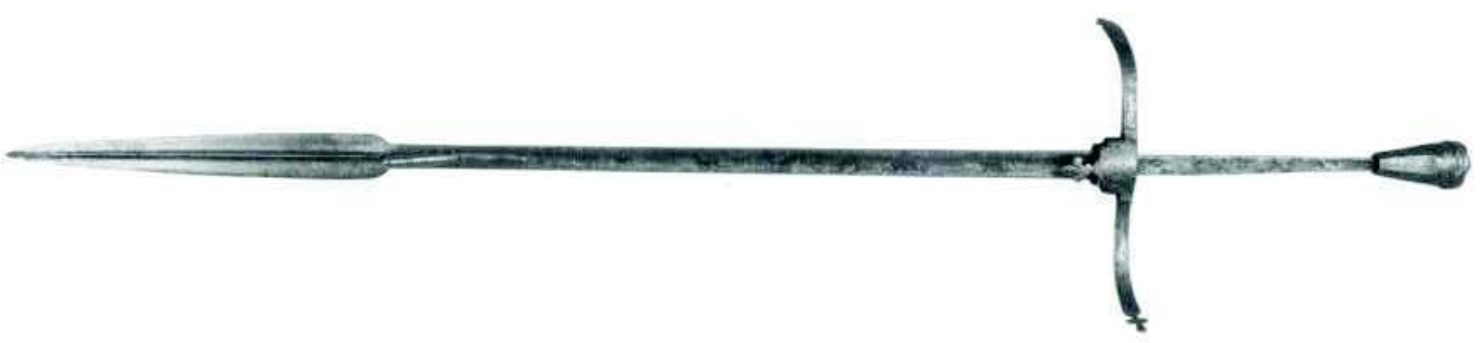

Figure 3 : Épée de chasse attribuée à René d’Anjou. (C) Firenze, Museo Nazionale del Bargello, inv. Carrand 1669

Il est toutefois certain qu'il ne s'agit pas d'une épée de chasse dont la typologie 3 offre de relatives similitudes. Ces armes d'estoc (voir fig. 3) s'utilisent comme des lances et possèdent souvent une pointe renforcée en feuille de laurier, avec des mécanismes d'arrêt, comme par exemple l'épée de chasse de René d'Anjou (voir fig. 3) 4 .

Contrairement à l'épée d'armes présentée ici, elles ne s'emploient que d'estoc et si la morphologie de ces armes permet de saisir la lame, c'est bien la seule analogie. En effet, l'épée de Vienne peut s'utiliser aussi de taille et ne correspond pas strictement aux critères des épées cynégétiques, sa forme se rapprochant plus de celle d'une épée de guerre5.

Il s'agit pas non plus d'une épée de "munition ", à disposition des citoyens pour la défense des villes, qui aurait permis de saisir la lame afin «de mieux parer les coups à deux mains » ou d'utiliser l'épée " comme une massue percuter l'adversaire avec le pommeau ${ }^{6}$.

Malgré ces incertitudes, la majeure partie de la littérature critique s'accorde à faire un lien entre cette épée et les livres de combat, sans plus de précisions utiles7. Ces livres décrivant les techniques de combat singulier, avec et sans armure, à pied et à cheval, offrent un corpus d'images important permettant notamment d'étudier les représentations de l'armement spécifique $^{8}$. Ainsi, je propose ici de livrer au lecteur une approche technique de l'usage de ce type d'épée, tel que décrit dans les livres de combat.

Identifier un type d'armes à partir d'un corpus iconographique pose déjà de nombreuses questions (validité des représentations, identification des écoles de dessin, correspondance avec des objets conservés, etc.). Les associer à des pratiques telles que des duels en armure au cours de la période de rédaction de ces manuscrits (XVe-XVIe siècle) nécessite en plus une prudence particulière dans le choix des sources et des méthodes d'analyse. La place manque ici pour faire les développements méthodologiques nécessaires et l'inventaire des différents contextes historiques et géographiques qui développent chacun des particularismes dans l'évolution des duels.

Je vais toutefois développer à partir de deux postulats. Le premier est le lien entre les livres de combat et la pratique du combat singulier, à plaisance ou à outrance9, en particulier le duel judiciaire, dans le Sud de l'Empire, le duché de Bourgogne et le Nord de l'Italie, de la première moitié du XVe siècle au premier tiers du XVIe siècle. Le second est le regroupement de différentes formes de combats singuliers (le duel judiciaire, le duel en tournoi et le duel d'honneur ${ }^{10}$ ) dans un même ensemble logique qui converge dans la pratique. En effet, ces différents types de combat sont tous régis par des règles, arbitrés et regardés par une audience. 
Chacun de ces combats donnaient lieu à une préparation souvent importante dans le temps, ainsi que l'achat ou la mise à disposition d'un matériel spécifique, en particulier pour l'armement. Dans son traité consacré aux tournois, Antoine de la Sale ${ }^{11}$ appelle ce type d'affrontement « combat des quatre pointes ». Il présente les quatre armes utilisées en son temps dans les affrontements

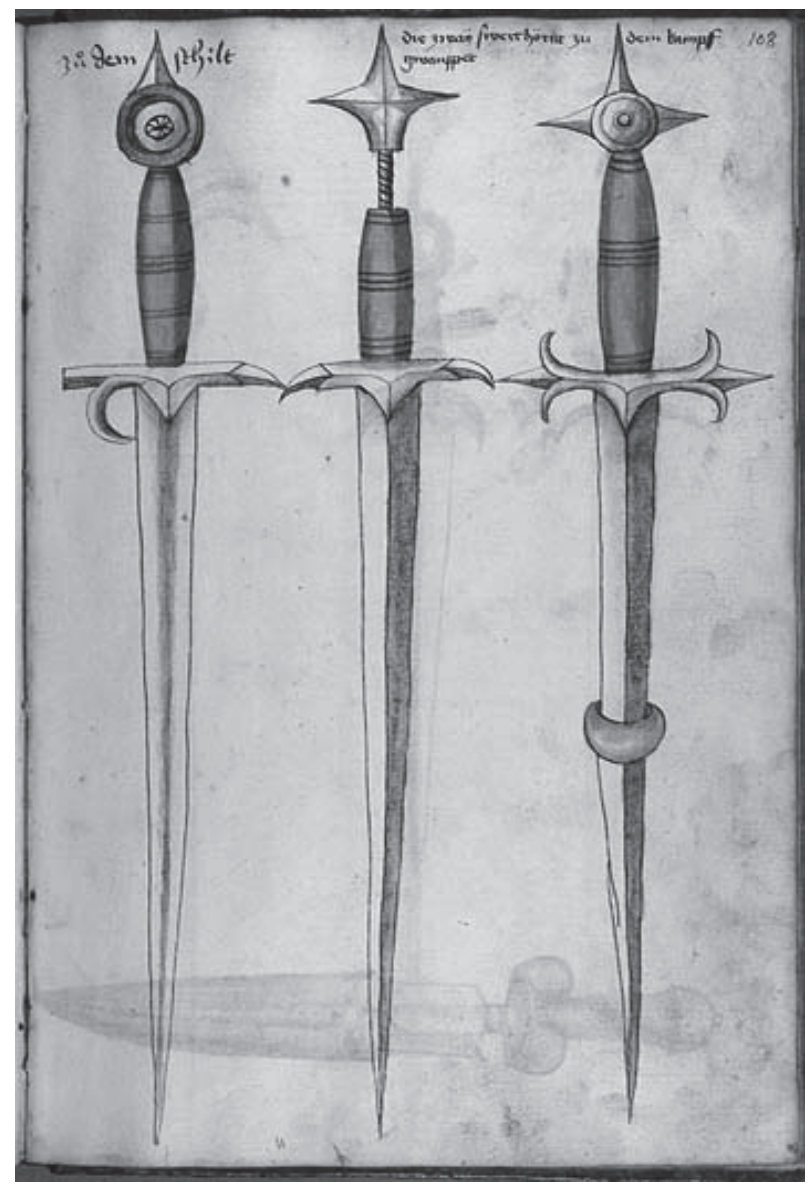

Figure 4: Hans Talhoffer, Alte Armature und Ringkunst, 1459. (C) Det Koneglige Bibliothek, Kopenhagen, Thott 290 $2^{\circ}, f^{\circ} 107 v$. normés à pied en armure, à savoir: la lance, la hache, l'épée et la dague. Cette tétralogie correspond exactement au répertoire technique décrit dans les livres de combat.

«Saisis l'épée avec la main droite par la poignée et avec la gauche attrape le milieu de la lame. Rapproche-toi de ton adversaire de manière à ce qu'il

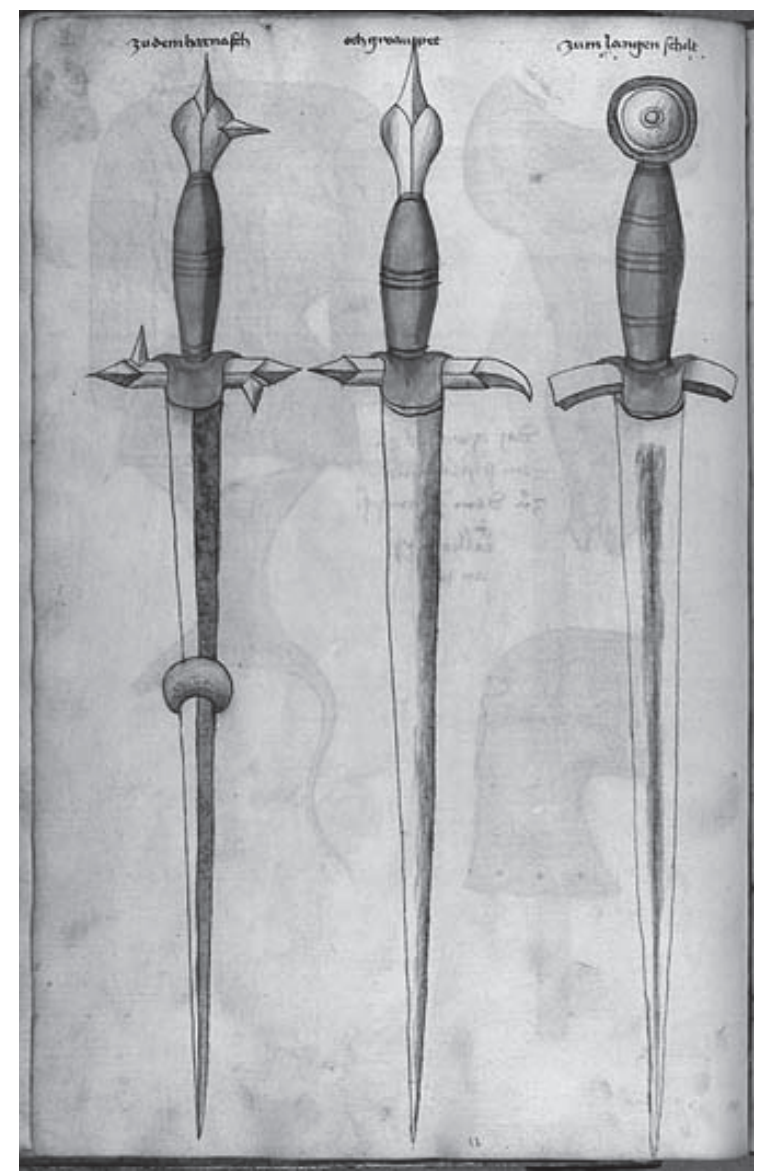

Figure 5: Hans Talhoffer, Alte Armature und Ringkunst, 1459. (C) Det Koneglige Bibliothek, Kopenhagen, Thott 290 $2^{\circ}, f^{\circ} 108 r$. 
doive estoquer ou frapper. Alors prends les devant, attaque de manière menaçante et reste proche. ${ }^{12}$

Par ces conseils généraux s'ouvre le traité d'André Lignitzer, compilé en premier dans un manuscrit daté de $1452^{13}$. Ce type de saisie permet en effet à un combattant en armure de transformer son épée, dont les coups de taille sont rendus moins performants par l'armement défensif adverse, en une lance de manière à percer les faiblesses de l'armure ${ }^{14}$. L'ensemble des techniques codifiées dans les sections consacrées au combat en armure à pied dans les livres de combat proposent cette tenue d'épée, nommée "demi-épée » ou "épée raccourcie».

Ces épées d'armes, spécifiques au combat à pied en armure, parfois dénommées estocs dans les sources secondaires, répondent à deux besoins : la protection de la main avancée qui saisit la lame et l'amélioration des qualités vulnérantes de certaines parties de l'arme (pommeau et garde). Différentes représentations de ces armes se trouvent dans le corpus des livres de combat : épées classiques améliorées simplement par l'ajout d'une rondelle sur la lame, épées munies de pointes et de crochets au pommeau et à la garde ${ }^{15}$, épées complexes telles que peintes dans le livre de 1459 d'Hans Talhoffer (voir fig. 4 et 5).

Un des manuscrits du groupe Gladiatoria ${ }^{16}$ suggère même que certaines parties étaient amovibles: une technique propose de dévisser le pommeau pour le jeter sur l'adversaire afin de le surprendre ${ }^{17}$. Cette information relativement surprenante révèle d'ailleurs une faiblesse structurelle relative de l'arme, contrairement à une construction classique qui voit le pommeau riveté à la soie de la lame. Ceci permet notamment d'envisager que si peu de ces épées d'armes aient survécu jusqu'à nos jours. De nombreux exemples démontrent que des pièces d'armures spécifiques étaient réalisées pour ce type d'événement, difficilement utilisables dans un autre contexte, ou alors transformées après l'événement. Il est tout à fait concevable que ces épées aient eu un caractère « consommable ${ }^{18}$.

Fiore dei Liberi et son continuateur Filippo Vadi sont les seuls à nous en donner une description précise (voir fig. 6, 7 et 8) :

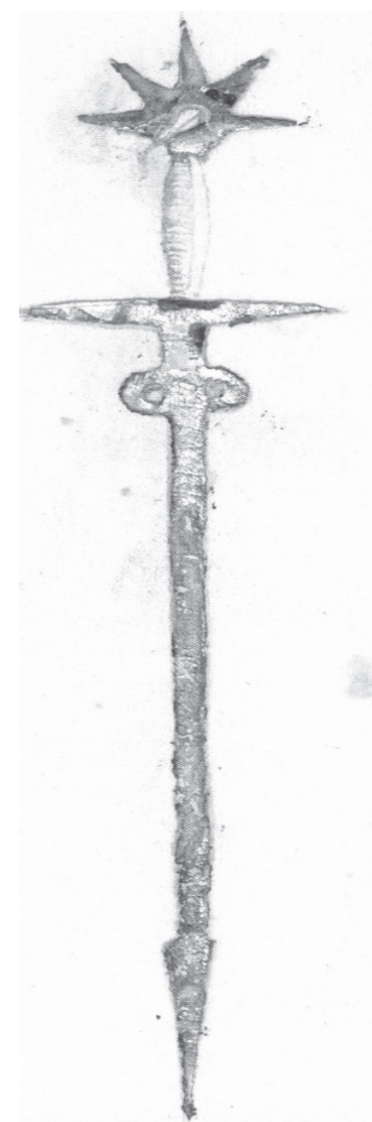

Figure 6 : Fiore dei Liberi, Fior di Battaglia, 1410. Los Angeles, Getty Museum, Ms Ludwig XV 13, $f^{\circ} 35 r$

"Cette épée est utilisée comme épée et comme lance et non pour couper... Et la rondelle qui se trouve après la garde doit pouvoir bouger jusqu'à un somesso $^{19}$ avant la pointe et pas plus et la garde doit être bien trempée et avoir 
de bonnes pointes et le pommeau doit être lourd. Et ces pointes doivent être trempées et bien pointues. Et l'épée doit être plus lourde en arrière qu'elle le sera devant (à vérifier). Et doit être de poids de 5 à 7 livres et suivant sa taille et sa force, selon la manière dont il veut être équipé. » ${ }^{20}$ (fig. 6)

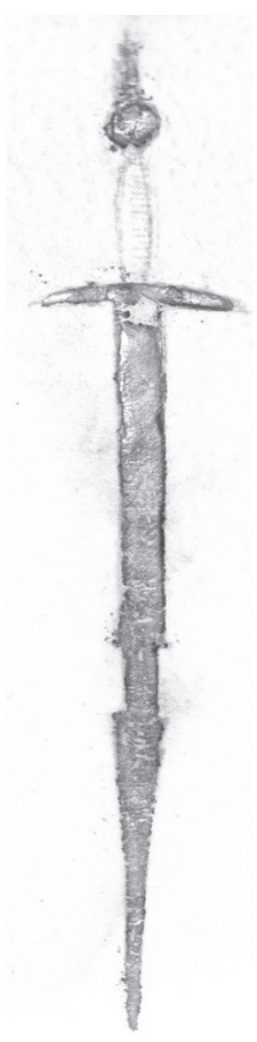

Figure 7 : Fiore dei Liberi, Fior di Battaglia, 1410. Los Angeles, Getty Museum, Ms Ludwig XV 13, $f^{\circ} 35^{r}$

«Cette autre épée peut tailler partout. Sauf que depuis la garde jusqu'à la pointe, dans les deux parties du milieu, un tiers de la pointe ne doit pas tailler, de manière à ce qu'une main avec un gantelet puisse tenir dedans. Et elle doit être aussi bonne de taille que d'estoc. La garde doit être bien trempée et pointue et le pommeau doit être lourd » (fig. 7)

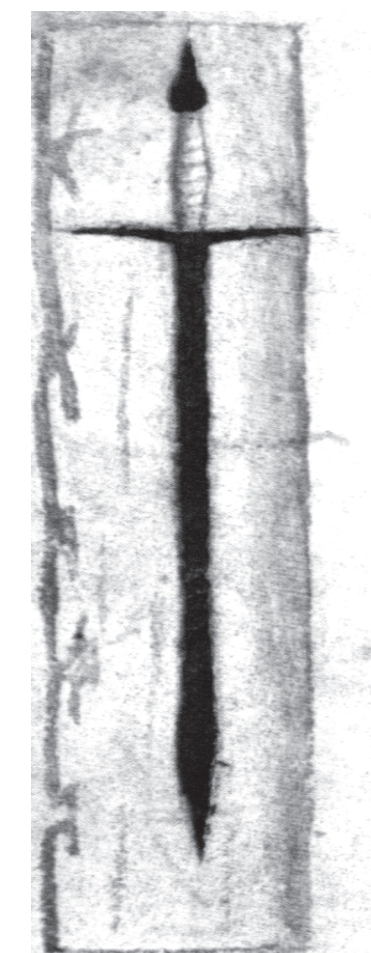

Figure 8 : Philippo Di Vadi, Arte Gladiatoria Dimicandi, 1482-1487. (C) Roma, Biblioteca dell'Academica Nazionale dei Lincei e Corsiniana, Codex 1342, $f^{\circ} 27 v$

«L'épée de combat en armure doit être des dimensions décrites cidessous, c'est-à-dire : sa longueur doit être telle que le pommeau arrive sous l'aisselle ; elle doit être [aiguisée pour] couper 4 doigts à partir de la pointe et sa poignée doit être d'un pan. La garde doit être de la même longueur que la poignée et doit être pointue de chaque 
côté; le pommeau doit être aussi pointu, de manière à pouvoir frapper avec chacune de ces parties. ${ }^{21}$ (fig. 8)

Le lecteur aura certainement remarqué les analogies entre la deuxième épée décrite par Fiore dei Liberi (voir fig. 7) et celle qui fait l'objet de ce petit article. La poignée centrale permet la prise en gantelet et l'arme peut être utilisée de taille et d'estoc. Voici donc attestées les raisons de la présence de cette partie centrale sur la lame.

Les liens entre les illustrations de ces armes spécifiques, la littérature technique codifiant leur usage et les objets conservés, tels que présentés ici nécessitent toutefois une étude interdisciplinaire plus large, prenant en compte plus de témoins. Ce travail reste encore à mener et nécessitera une enquête plus large dans les fonds muséaux à la recherche d'autres spécimens d'épée d'armes.

Je conclus ici en livrant une technique commentée, typique du maniement de cette arme. La pièce est tirée du traité de Martin Hundsfeld ${ }^{22}$, dans la partie qui traite du combat à l'épée en armure à pied : «Brise le placement de la pointe au visage ou ailleurs de la manière suivante : $[\ldots]$ »

Il s'agit d'une contre technique à une estocade dans le visage ou dans une autre cible primaire comme la gorge et l'aisselle. Il est possible de survivre à un coup de pointe placé, passant les défenses dans les faiblesses ${ }^{23}$, pour autant que l'angle d'attaque, ainsi que le déplacement corporel suite au choc et à l'insertion de l'arme, fassent que l'épée adverse soit " enferrée », c'est-à-dire coincée dans les pièces d'armure ou ayant buté contre. Cela implique néanmoins une blessure grave, mais pas forcément ou immédiatement mortelle suivant l'angle final de l'estocade. L'adversaire est un instant dans une position relativement précaire, son arme étant bloquée. La fenêtre temporelle pour placer une contre technique est courte, car soit il placera sa pointe avec un autre angle, assurant sa position et infligeant des dégâts plus importants, soit il retirera son épée pour placer une seconde estocade dans la même faiblesse ou dans une autre, entre autres choix possibles de techniques applicables.

« Estoque-le dessous, dans la main qu'il a placée sur la lame à l'avant et conduis-le hors du cercle. » . La première solution est de percer la paume de la main. Ce faisant, en poussant l'estocade en demiépée, l'auteur de la contre technique crée un déséquilibre, ainsi qu'une forme de prise de soumission qui permet de "conduire" l'adversaire hors du "cercle". Cette action, dans nombre de combats normés, qu'ils soient à outrance ou à plaisance, signifie l'interruption de l'affrontement.

«Ou estoque-le au-dessus de sa main avancée et appuie l'épée avec le pommeau vers le bas et placelui ta pointe. ». Cette action permet, en utilisant son arme comme levier, de faire lâcher l'arme adverse tout en plaçant sa pointe dans une position favorable pour une estocade dans ses faiblesses.

«Ou dévie-le entre tes deux mains. Pousse le pommeau vers sa gorge, marche avec le pied droit derrière son gauche et projette-le par-dessus. ". Cette action implique un déplacement vers la droite qui permet de modifier l'angle de son corps par rapport à l'attaque adverse en forçant avec une poussée de son arme des deux mains, pommeau vers le haut. Suivant où l'arme adverse est enferrée, cela permet de s'en libérer, ou du moins d'atténuer ses capacités vulnérantes. La suite de la technique est une projection classique par-dessus la jambe en s'aidant du pommeau qui vient se placer sous la gorge adverse.

Le texte livre cinq autres contre techniques à la suite de celles-ci, avant de poursuivre avec d'autres pièces. Les trois décrites ci-dessus, illustrent de manière représentative la finalité des techniques consacrées au combat en armure à pied codifiées dans les livres de combat: une se termine par une action qui provoque la transgression d'une norme ou d'une règle ; une autre par une attaque mortelle ou incapacitante; une dernière par une projection qui permet soit d'interrompre le combat 
(transgression d'une norme), soit de placer l'adversaire dans une position précaire pour permettre un achèvement ou une soumission.

${ }^{1}$ En l'état actuel des connaissances, il n'existe qu'une seule autre épée possédant ces caractéristiques, conservée au Kunsthistorisches Museum (Hofjagd- und Rustkammer) à Vienne, pièce $\mathrm{n}^{\circ} \mathrm{A} 90$. Toutefois mes recherches sur les livres de combat et deux autres spécimens examinés me permettent de considérer ces armes comme appartenant à une typologie particulière correspondant à un usage avéré.

2 THOMAS Bruno et GAMBER Ortwin, Katalog der Leibrüstkammer: Der Zeitraum von 500 bis 1530, Wien, Kunsthistorisches Museum, 1976, p.66. SCALINI Mario (éd.), $A$ Bon Droyt : Spade Di Uonimi Liberi Cavalieri E Santi = Épées D’hommes Libres, Chevaliers Et Saints, Milano, Silvana, 2007, p. 212. NIEHOFF Franz (Hrsg.), Ritterwelten im Spätmittelalter, Landshut, Museen der Stadt Landshut, 2009, p. 233-234 et HUYNH Michel (éd.), L'épée: Usages, Mythes Et Symboles: [exposition], Paris, Musée De Cluny - Musée National Du Moyen Age, 28 Avril - 26 Septembre 2011, Paris, Réunion des Musées nationaux - Grand Palais, 2011, p.120.

3 Voir notamment : BORD Lucien-Jean et MUGG Jean-Pierre, $L a$ chasse au Moyen Âge: Occident latin, VIe-XVe siècle, Aix-enProvence, Le gerfaut, 2008, p.202-218.

4 Pièce 35 du catalogue HUYNH, 2011, op. cit.. Commentaires sur son usage aux pages 42,43 et 108 .

5 OAKESHOTT Ewart, Records of the Medieval Sword, Woodbridge, Boydell Press, 1991.

${ }^{6}$ SCALINI, 2007, op. cit., dans la description de l'objet 49, p. 212.

7 À l'exception de catalogue HUYNH, 2011, op. cit., qui précise le lien avec le combat en armure - Harnischfechten - dans son commentaire.

${ }^{8}$ La majeure partie de ces livres ont fait l'objet d'un catalogue : LENG Rainer, FRÜHMORGEN-VOSS Hella et al., Katalog der deutschsprachigen illustrierten Handschriften des Mittelalters Band 4/2, Lfg. 1/2: 38: 38. Fecht- und Ringbücher, München, C.H. Beck, 2009. Pour des articles de fond sur le sujet, voir: JAQUET Daniel (éd.), L'art chevaleresque du combat: le maniement des armes à travers les livres de combat (XIVe XVIe siècle), Neuchâtel, Alphil, 2013.

9 Dès le XIVe siècle, il est de coutume de faire la distinction entre un combat à mort (ou avec des armes létale), dit "à outrance" et un combat ludique (ou avec des armes sécurisée), dit "à plaisance". JAQUET Daniel, "Combattre à plaisance ou à outrance? Le combat en armure à pied d'arpès les textes fondateurs », dans JAQUET (éd.), 2013, op. cit., p. 153-171.

${ }^{10}$ Voir à ce sujet CAVINA Marco, Il Sangue Dell'onore: Storia Del Duello, Roma, Laterza, 2005, 327 p.

${ }^{11}$ LEFEVRE Sylvie, Antoine de la Sale. La fabrique de l'oeuvre et de l'écrivain. Suivi de l'édition critique du "Traité des anciens et des nouveaux tournois", Genève, Droz, 2006.

${ }^{12}$ André LIGNITZER, épée raccourcie, $1452, \mathrm{f}^{\circ} 73 \mathrm{r}$. Publié dans HAGEDORN Dierk, Peter von Danzig: Transkription und Übersetzung der Handschrift 44 A 8, Torsten Verhülsdonk, VSBOOKS, 2009. Traduction de l'auteur.

${ }_{13}$ Biblioteca dell'Accademia Nazionale dei Lincei e Corsiniana, Roma, Codex 44 A 8.

${ }^{14}$ À savoir : la vision, la gorge, les aisselles et les parties génitales. D'autres cibles secondaires apparaissent dans les sources: la pliure du coude, l'intérieur du genou et la paume de la main.

15 ZABINSKI Grzegorz et WALCZAK Bartlomiej mentionnent brièvement ces armes qu'ils dénomment "judicial swords" dans leur ouvrage Codex Wallerstein. A Medieval Fighting Book from the fifteenth Century, Boulder, Paladin Press, 2002, p. 4 , où ils listent les occurrences des illustrations de ces armes dans le manuscrit étudié et mentionnent la planche de Hans Talhoffer illustrée ci-dessous. Il faut rajouter de nombreuses occurrences dans les manuscrits de Jorg Wilhalm, Paulus Hector Mair et trois des manuscrits de Hans Talhoffer.

${ }^{16}$ Groupe de manuscrits produits dans la première moitié du XVe siècle. Voir LENG, 2009, op. cit., p. 22-34

17 «Remarque la douzième [technique], lorsque tu veux le surprendre. Alors prends ta lance et ton épée ensemble sous le bras et dévisse le pommeau de ton épée et jette-le violemment sur lui et rentre-lui dedans après le lancer [...]». LORBEER Carsten (Hrsg), Transkription der Hs. Wien, Kunsthistorisches Museum, KK5013, Gesellschaft für pragmatische Schriftlichkeit, 2006, $\quad$ 9, <http://www.pragmatischeschriftlichkeit.de/transkription/trans_KK5013_w_e.pdf>, consulté le 12.02.11. Traduction de l'auteur.

${ }^{18}$ Je pointe ici les pistes d'un travail de recherche sur le sujet qui reste à mener.

19 Unité de mesure correspondant à une main.

20 Texte édité par RUBBOLI Marco et CESARI Luca, Flos duellatorum : manuale di arte del combattimento del XV. secolo, Rimini, Il Cerchio, 2002, p. 202. Traduction de l'auteur. ${ }^{21}$ Texte édité par PORZIO Luca and MELE Gregory, Arte gladiatoria dimicandi: 15th century Swordsmanship of Master 
Filippo Vadi, Union City; Woodbridge, Chivalry Bookshelf; Boydell \& Brewer, 2003. Traduction de l'auteur.

${ }^{22}$ Édité dans HAGEDORN, 2008, op. cit. , f ${ }^{\circ}$ 89r-89v. Traduction de l'auteur.

23 Les faiblesses décrites sont couvertes par un vêtement composé de plusieurs couches de tissus avec présence possible de cuir, renforcé par des goussets de maille. La section carrée de ces armes spécifiques offrent d'ailleurs l'avantage de pouvoir faire sauter les rivets et écarter les anneaux sous la pression d'une estocade portée. 


\section{BIBLIOGRAPHIE}

BORD Lucien-Jean et MUGG Jean-Pierre, $L a$ chasse au Moyen Âge: Occident latin, VIe-XVe siècle, Aix-en-Provence, Le gerfaut, 2008.

CAVINA Marco, Il Sangue Dell'onore: Storia Del Duello, Roma, Laterza, 2005.

HAGEDORN Dierk, Peter von Danzig: Transkription und Übersetzung der Handschrift 44 A 8, Herne, VS-BOOKS, 2009.

HUYNH Michel (éd.), L'épée: Usages, Mythes Et Symboles: [exposition], Paris, Musée De Cluny Musée National Du Moyen Age, 28 Avril - 26 Septembre 2011, Paris, Réunion des Musées nationaux - Grand Palais, 2011.

JAQUET Daniel (éd.), L'art chevaleresque du combat: le maniement des armes à travers les livres de combat (XIVe - XVIe siècle), Neuchâtel, Alphil, 2013.

LEFEVRE Sylvie, Antoine de la Sale. La fabrique de l'oeuvre et de l'écrivain. Suivi de l'édition critique $d u$ "Traité des anciens et des nouveaux tournois", Genève, Droz, 2006.

LENG Rainer, FRÜHMORGEN-VOSS Hella et al., Katalog der deutschsprachigen illustrierten Handschriften des Mittelalters Band 4/2, Lfg. 1/2: 38: 38. Fecht- und Ringbücher, München, C.H. Beck, 2009.

LORBEER Carsten (Hrsg), Transkription der Hs. Wien, Kunsthistorisches Museum, KK5013, Gesellschaft für pragmatische Schriftlichkeit, 2006, p. 9 : $\quad$ http://www.pragmatischeschriftlichkeit.de/transkription/trans_KK5013_w_ e.pdf, consulté le 12.02.11.

NIEHOFF Franz (Hrsg.), Ritterwelten im Spätmittelalter, Landshut, Museen der Stadt Landshut, 2009.
OAKESHOTT Ewart, Records of the Medieval Sword, Woodbridge, Boydell Press, 1991.

PORZIO Luca and MELE Gregory, Arte gladiatoria dimicandi: 15th century Swordsmanship of Master Filippo Vadi, Union City; Woodbridge, Chivalry Bookshelf; Boydell \& Brewer, 2003.

RUBBOLI Marco et CESARI Luca, Flos duellatorum : manuale di arte del combattimento del XV. secolo, Rimini, Il Cerchio, 2002.

SCALINI Mario (ed.), A Bon Droyt: Spade Di Uonimi Liberi Cavalieri E Santi = Epées D'hommes Libres, Chevaliers Et Saints, Milano, Silvana, 2007.

THOMAS Bruno und GAMBER Ortwin, Katalog der Leibrüstkammer: Der Zeitraum von 500 bis 1530, Wien, Kunsthistorisches Museum, 1976, p.66.

ZABINSKI Grzegorz and WALCZAK Bartlomiej, Codex Wallerstein. A Medieval Fighting Book from the fifteenth Century, Boulder, Paladin Press, 2002. 